\title{
Impact of the Particle Oxidation Catalyst on the Particle Number Emission of the Diesel Engine
}

\author{
Jiaqiang $\mathrm{Li}^{1, \mathrm{a}}$, Chao $\mathrm{He}^{1, \mathrm{~b},{ }^{*}}$, Yong Wang ${ }^{2, \mathrm{c}}$, and Yanyan Wang ${ }^{1, \mathrm{~d}}$ \\ ${ }^{1}$ School of Mechanical and Traffic Engineering, Southwest Forestry University, Kunming 650224, \\ China. \\ ${ }^{2}$ Yunnan Yuntong Judicial Expertise Center, Kunming 650224 China.

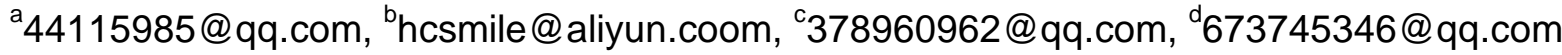

Keywords: Particle oxidation catalyst; Particle number emission; Size distributions; Diesel engine

Abstract: Due to their adverse health effects and their abundance in urban areas, diesel exhaust ultrafine particles caused by the aftertreatment devices have been of great concern in the past years. An experiment of particles number emissions was carried out on a high-pressure, common rail diesel engine with particle oxidation catalyst (POC) to investigate the impact of POC on the number emission characteristics of particles. The results indicated that the conversion rates of POC is $32.4 \%-95.9 \%$ with the average value of $61 \%$. The conversion rate of POC decreased with the increasing particle diameter and POC has a good reduction effect on the nucleation mode particles. The geometric number mean diameters of particles downstream POC is bigger than those upstream POC, which means that POC has a higher conversion rate on the nucleation mode particles.

\section{Introduction}

Due to their adverse health effects and their abundance in urban areas, diesel exhaust particles (DEP) have been of great concern in the past years ${ }^{[1]}$. The traditional method to measure particle emissions for type approval tests is gravimetric analysis and the detection limit of this method will be already achieved with the Euro IV limit. Many pathophysiological researches suggest that exposure to DEP, especially ultrafine particles, is associated with increased respiratory disease, cardiopulmonary mortality and other potential health effects ${ }^{[2]}$. Over the past few years, researches have begun to address the questions of the number concentration of $\mathrm{DEP}^{[3,4]}$ and regulations have been made to the number emissions of DEP in Euro V and VI emission standards.

The aftertreatment devices of diesel engines can reduce the exhausted pollutants, however, some researchers have reported that they can improve the generation of ultrafine particles. Maricq et al. studied the impact of DOC and sulfur content on the size distribution of DEP ${ }^{[5]}$. The results indicated that the nucleation mode particles would increase and the bimodal size distribution would appear only when the DOC and diesel with high sulfur were used together. Otherwise, the mono-modal distribution of the accumulation mode would occur. Biswas et al. investigated the size distributions, volatility, and agglomeration of particles from a heavy-duty diesel engine with DPF and SCR ${ }^{[6]}$. The advanced aftertreatment can decrease the mass of PM by $90 \%$, but the number emissions of particles did not decrease on the stead modes and the nucleation mode particles increased. In this study, an experiment was carried out to investigate the number emission characteristics of particles emitted by the diesel engine with particle oxidation catalyst (POC).

\section{Experimental setup}

Test devices The test engine is a direct injection, high pressure common-rail, turbocharged diesel engine (WeiChai Power WP5, China), whose characteristics are shown in table 1. The engine with POC was tested on an engine test bench based on an AC dynamometer (Schenck HT350, Germany) with emission measurement system. An engine exhaust particle sizer (EEPS 3090, TSI, USA) was used for the investigation of particle matter (PM). To prevent the particle condensation and nucleation, two stages of ejector diluter (ED) upstream EEPS were used for sampling from raw exhaust stack 
(Burtscher, 2005). The fuel used in this study was locally available commercial low sulfur (50 ppm) diesel.

Table 1 Specification of the test engine

\begin{tabular}{lc}
\hline Parameter & Feature/Size \\
\hline Engine type & 4-stroke, 4-cylinder, in-line \\
Bore $\times$ Stroke $(\mathrm{mm})$ & $108 \times 10$ \\
Displacement $(\mathrm{L})$ & 4.76 \\
Compression ratio & 18 \\
Fuel system & High pressure common rail \\
Max. Power $(\mathrm{kW} @ \mathrm{r} / \mathrm{min})$ & $132(2300 \mathrm{r} / \mathrm{min})$ \\
Max. Torque $(\mathrm{Nm} @ \mathrm{r} / \mathrm{min})$ & $650(1200-1600 \mathrm{r} / \mathrm{min})$ \\
\hline
\end{tabular}

Measurement methods The exhaust pollutant, temperature and pressure measurements were performed at the upstream and downstream of the after-treatment device. The results of emissions, fuel consumption and relevant engine parameters were recorded by the dynamometer control system. The stationary operation points (OPs), so called steps-tests were performed. The engine was operated at $10 \%, 20 \%, 30 \%, 40 \%, 50 \%, 60 \%, 70 \%, 80 \%, 90 \%$ and $100 \%$ of full load at seven engine speeds $(1000,1200,1400,1600,1800,2000$ and $2300 \mathrm{r} / \mathrm{min})$. All operation points were performed with a warm engine and for each research task always in the same sequence.

A portion of the exhaust gas emitted from the engine was extracted from the tailpipe and quickly diluted by two ejector dilutors (Dekati Ltd.) in series. The dilution air is preconditioned to remove the water and volatile organic compound (VOC). The dilution air and diluted exhaust in the first dilutor are heated to $200^{\circ} \mathrm{C}$ in order to prevent condensation. The second ejector dilutor is at room temperature to cool the sample prior to distribution into the EEPS. The dilution ratio during test procedure is around 64, which essentially inhibits all post-dilution particle dynamics such as coagulation and adsorption ${ }^{[7]}$. The dilution factor (DF) was found with the following expression:

$$
D F=\left(C_{e}-C_{a}\right) /\left(C_{t}-C_{a}\right)
$$

where $\mathrm{C}_{\mathrm{e}}, \mathrm{C}_{\mathrm{t}}$ and $\mathrm{C}_{\mathrm{a}}$ respectively denote the $\mathrm{CO}_{2}$ concentration in the engine-out exhaust gas, in the dilution tunnel and in the air.

\section{Results and discussions}

The total particle number emissions of DEP Figure 1 show the total number emissions of DEP unstream and downstream POC. The total particle number emissions is about $1.5 \times 10^{6}-3.8 \times 10^{7} 1 / \mathrm{cm}^{3}$ upstream POC and $8.6 \times 10^{5}-5.7 \times 10^{6} 1 / \mathrm{cm}^{3}$ downstream POC. The emission peak of engine-out particles is at the heavy loads of medium speeds. At low engine speeds, the total number emissions of DEP increased with the increasing engine loads upstream POC. At high engine speeds, it decreased with the increasing engine loads. Downstream POC, the emission peak occurred at the low speed and heavy loads. At low speeds, the total number emissions of DEP increased with the increasing engine loads. At medium engine speeds, it decreased with the increasing engine loads. The conversion rates of POC on DEP is about 32.4\% 95.9\% with the average value of $61 \%$. At the medium engine speeds and high loads, the conversion rates of POC is high, however, they keep low at the low speeds and high speeds high loads.

The size distributions of DEP Figure 3 showed the size distribution of DEP upstream and downstream POC and the conversion rates of POC at $1400 \mathrm{r} / \mathrm{min}$. At the low load, the size distribution of particles is bimodal lognormal distributions upstream POC and the two peak concentrations are at the diameter of $8-12 \mathrm{~nm}$ and $52-80 \mathrm{~nm}$. Downstream POC, the size distribution is mono-modal, and the peak is at the diameter of 52-93nm. The conversion rate of POC decreased with the increasing particle diameter. The conversion rate decreased from $85 \%$ at the diameter of $6-11 \mathrm{~nm}$ to $40 \%$ at the diameter of $107-124 \mathrm{~nm}$. At the full load, the maximum number concentration appeared at the diameter of 6-22nm, which belongs to the nucleation mode. POC has a good reduction effect on the nucleation mode particles, which decreased by $99 \%$. So the conversion rate of overall diameters is over $95 \%$. At 
other modes, the conversion rates of POC ranged from $54 \%$ to $68 \%$ with the average value of $60 \%$. The exhausted temperature at full load is higher than that at low load, which is advantageous for the oxidation of ultrafine particles, so the conversion rate is higher at the full load.

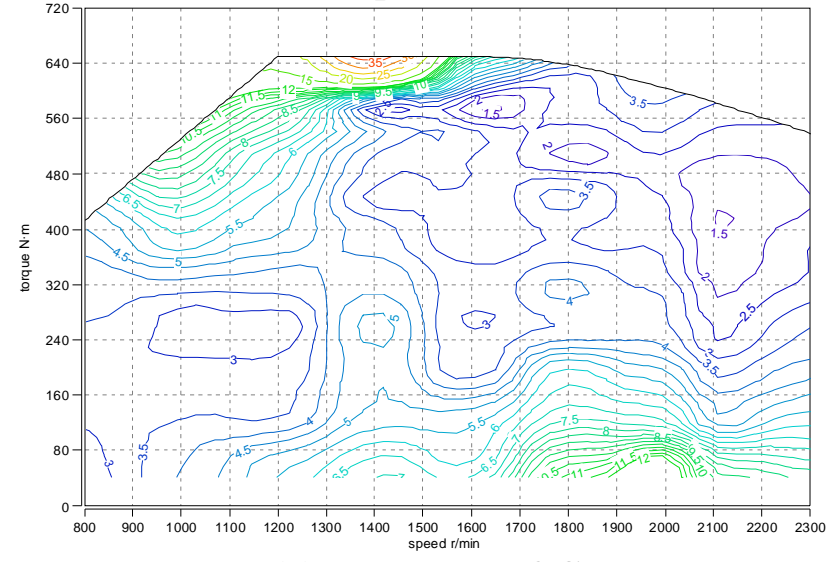

(a) Upstream POC

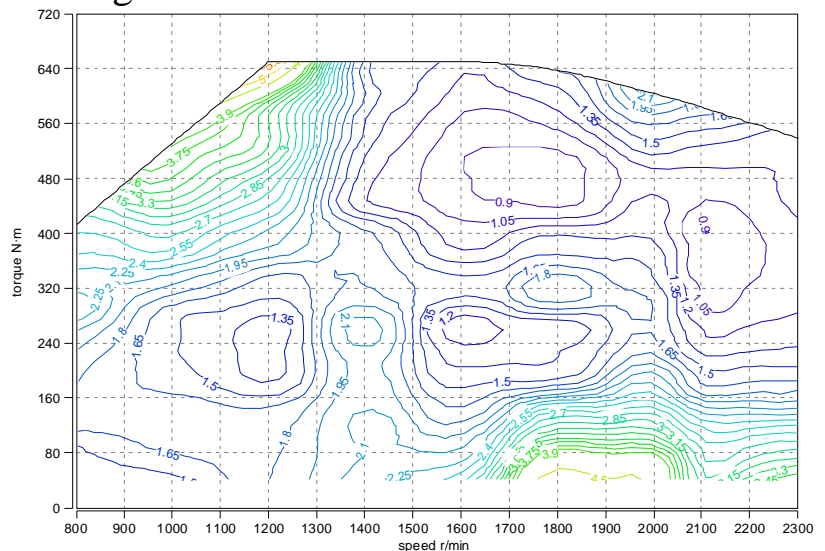

(b) Downstream POC

Fig. 1 The total number emissions of DEP unstream and downstream POC $\left(\times 10^{6} 1 / \mathrm{cm}^{3}\right)$.
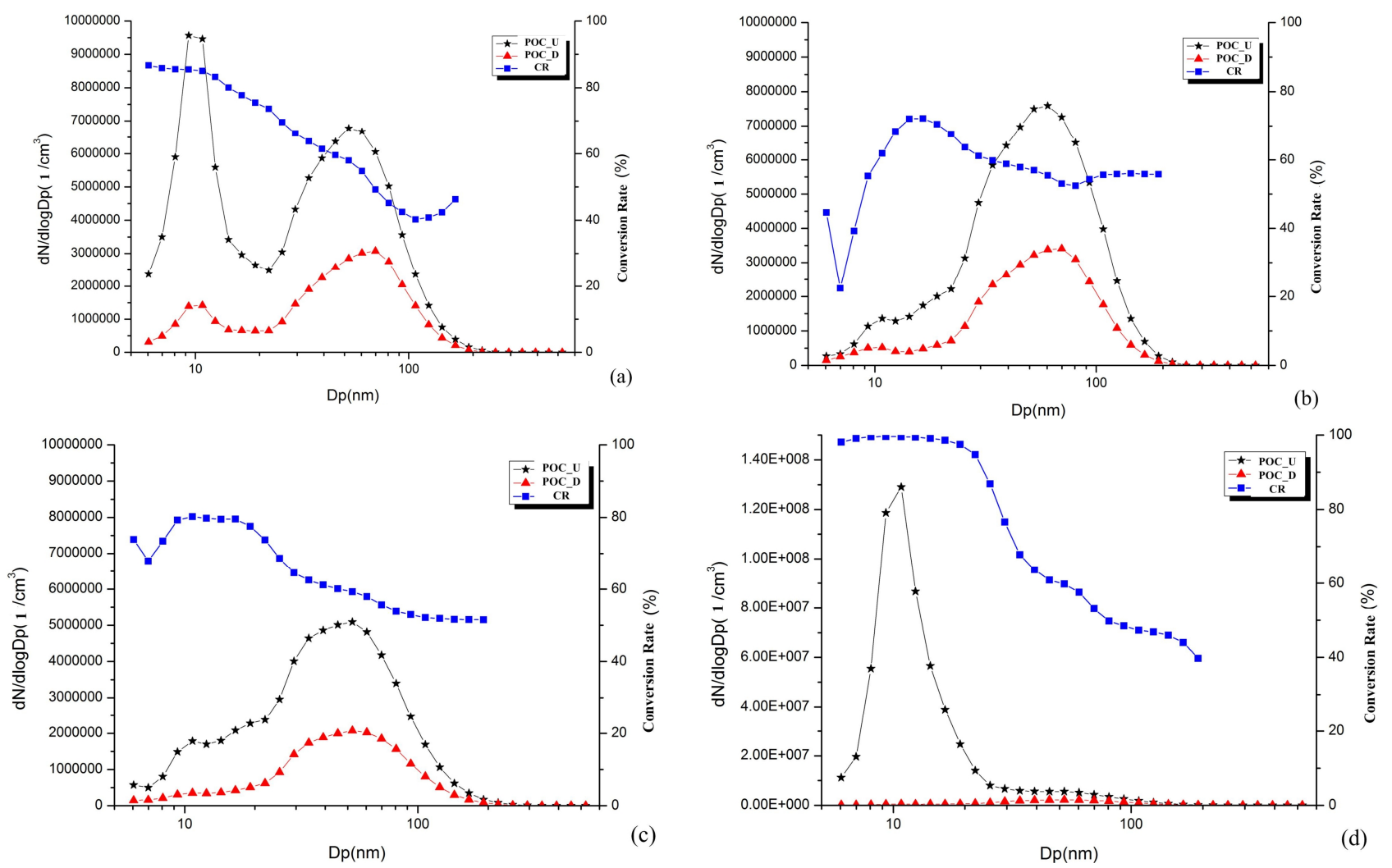

Fig. 2 the size distribution of DEP upstream and downstream POC and the conversion rates of POC at 1400 r/min. engine load: (a) 10\%, (b) 40\%, (c) 80\%, (d) 100\%. POC_U: upstream POC, POC_D: downstream POC; CR:conversion rate

Figure 3 showed the size distribution of DEP upstream and downstream POC and the conversion rates of POC at $2300 \mathrm{r} / \mathrm{min}$. At every load, the size distributions of particles are bimodal lognormal distributions upstream and downstream POC. The two peak concentrations upstream POC are at $6-12 \mathrm{~nm}$ and $34-52 \mathrm{~nm}$, while at the diameter of $8-12 \mathrm{~nm}$ and $34-60 \mathrm{~nm}$ downstream POC. The conversion rate of POC decreased with the increasing particle diameter at the low load, which is according with the change pattern at $1400 \mathrm{r} / \mathrm{min}$. The conversion rate decreased from $81 \%$ at the diameter of $6 \mathrm{~nm}$ to $30 \%$ at the diameter of $124-143 \mathrm{~nm}$. At the full load, the maximum number concentration appeared at the diameter of $40-60 \mathrm{~nm}$, which belongs to the accumulation mode. The conversion rates of POC ranged from $39 \%$ to $64 \%$ with the average value of $56 \%$, which is lower than that at $1400 \mathrm{r} / \mathrm{min}$. 


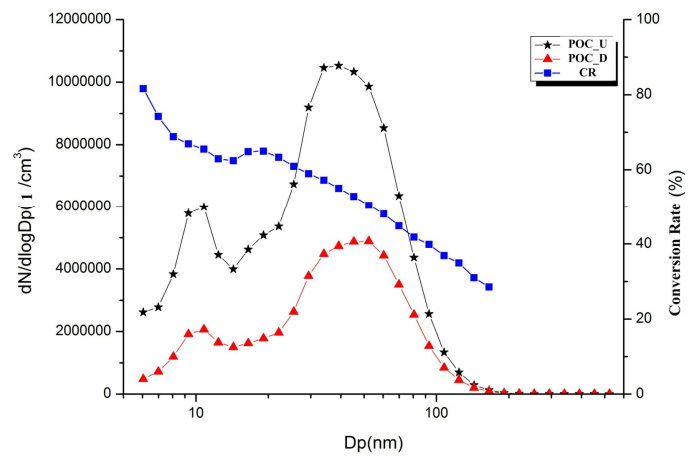

(a)

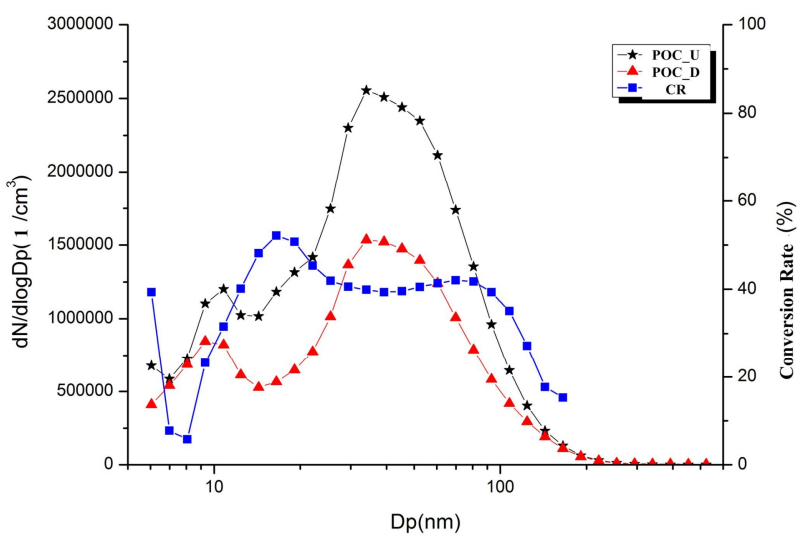

(c)
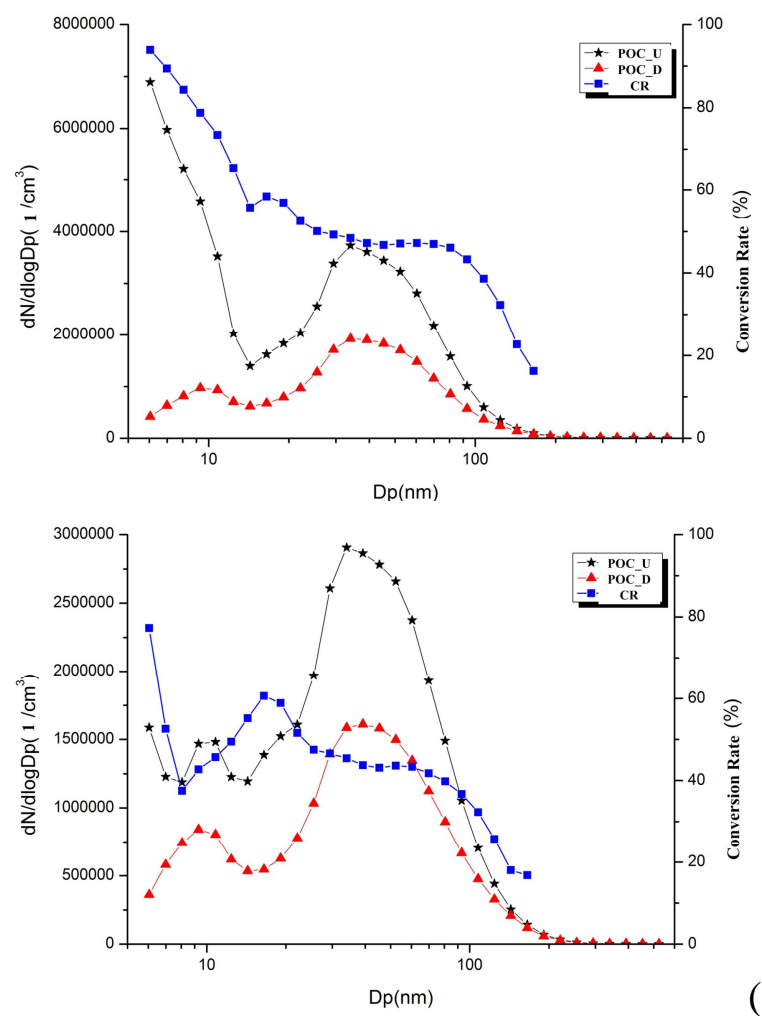

(b)

(d)

Fig. 3 the size distribution of DEP upstream and downstream POC and the conversion rates of POC at 2300 r/min. engine load: (a) 10\%, (b) 40\%, (c) 80\%, (d) 100\%. POC_U: upstream POC, POC_D: downstream POC; CR:conversion rate

GMD of exhausted particles Many literatures report that the experimentally measured size distribution of diesel exhausted particle can be fit to the lognormal form as following:

$$
\frac{d N}{d \ln D_{p}}=\frac{N}{\sqrt{2 \pi} \sigma} \exp \left[\frac{-\left(\ln D_{p}-\ln \mu\right)^{2}}{2 \sigma^{2}}\right]
$$

$\mathrm{N}$ is the number of particles, $\mu$ is the geometric number mean diameters (GMD) and $\sigma$ is the corresponding geometric standard deviation (GSD).GMD and GSD are two important parameters regarding particle size. Table 2 gave the GMD and GSD of particles upstream and downstream POC. It can be seen that the GMD downstream POC is bigger than that upstream POC. The GMD of engine-out particles are about $13-60 \mathrm{~nm}$, and the GMD of particle downstream POC are about 30-66nm. It means that POC has a higher conversion rate on the nucleation mode particles.

Table 2 The GMD and GSD of particles upstream and downstream POC

\begin{tabular}{cccccc}
\hline $\begin{array}{c}\text { Engine } \\
\text { speed } \\
(\mathrm{r} / \mathrm{min})\end{array}$ & $\begin{array}{c}\text { Engine } \\
\text { load }\end{array}$ & \multicolumn{2}{c}{ Upstream POC } & \multicolumn{2}{c}{ Downstream POC } \\
& $(\%)$ & $\begin{array}{c}\text { CMD } \\
(\mathrm{nm})\end{array}$ & GSD & $\begin{array}{c}\text { CMD } \\
(\mathrm{nm})\end{array}$ & GSD \\
\hline \multirow{3}{*}{1400} & 20 & 41 & 2.16 & 46 & 2.04 \\
& 40 & 46 & 1.95 & 49 & 1.96 \\
& 60 & 41 & 2.00 & 41 & 2.02 \\
& 80 & 37 & 2.07 & 44 & 1.97 \\
& 100 & 13 & 1.68 & 43 & 2.04 \\
& 20 & 28 & 2.15 & 32 & 2.09 \\
& 40 & 19 & 2.44 & 30 & 2.18 \\
& 60 & 21 & 2.47 & 30 & 2.21 \\
& 80 & 32 & 2.15 & 31 & 2.25 \\
& 100 & 29 & 2.26 & 32 & 2.25 \\
\hline
\end{tabular}




\section{Conclusions}

(1) The total particle number emissions of diesel engine is $1.5 \times 10^{6}-3.8 \times 10^{7} 1 / \mathrm{cm}^{3}$ upstream POC and $8.6 \times 10^{5}-5.7 \times 10^{6} 1 / \mathrm{cm}^{3}$ downstream POC. The conversion rates of POC is $32.4 \%-95.9 \%$ with the average value of $61 \%$.

(2) The conversion rate of POC decreased with the increasing particle diameter under the low load at $1400 \mathrm{r} / \mathrm{min}$ and $2300 \mathrm{r} / \mathrm{min}$, and POC has a good reduction effect on the nucleation mode particles. The conversion rates of POC at $2300 \mathrm{r} / \mathrm{min}$ is lower than that at $1400 \mathrm{r} / \mathrm{min}$.

(3) The geometric number mean diameters of particles downstream POC is bigger than those upstream POC, which means that POC has a higher conversion rate on the nucleation mode particles.

The GMD of engine-out particles are about 13-60nm, and the GMD of particle downstream POC are about 30-66nm.

\section{Acknowledgements}

This work was financially supported by the National Natural Science Foundation of China (NSFC, 51266015), Applied basic research project of Yunnan Province (2013FB052), Department of Education, Yunnan province (2013Z081) and Scientific Research Foundation of Southwest Forestry University (C14120).

* Corresponding author: Chao He, hcsmile@aliyun.com

\section{References}

[1] J. S. Lighty, J. M.Veranth, A. F. Sarofim, "Combustion aerosols: factors governing their size and composition and implications to human health," J. Air. Waste. Manage, vol. 50, pp.1565-1618, 2000. [2] C. A. Pope, D. W. Dockery, J. Schwartz, "Review of Epidemiological Evidence of Health-Effects of Particulate Air- Pollution," Inhal. Toxicol, vol. 7, pp. 1-18, 1995.

[3] M. M. Maricq, D. H. Podsiadlik, R. E Chase, "Size distributions of motor vehicle exhaust PM: a comparison between ELPI and SMPS measurements," Aerosol. Sci. Tech, vol. 33, pp. 239-260, 2000.

[4] D.B. Kittelson, W.F. Watts, J.P. Johnson, "On-road and laboratory evaluation of combustion aerosols-Part1: Summary of diesel engine results," J. Aerosol. Sci, vol. 37, pp. 913-930 , 2006.

[5] Maricq M M, Chase R E, Xu N, et al. The effects of the catalytic converter and fuel sulfur level on motor vehicle particulate matter emissions: Light duty diesel vehicles[J]. Environmental Science \& Technology, 2002, 36 (2) : 283-289.

[6] Biswas S, Hu S H, Verma V, et al. Physical properties of particulate matter (PM) from late model heavy-duty diesel vehicles operating with advanced PM and NOx emission control technologies[J]. Atmospheric Environment, 2008, 42 (22) : 5622-5634.

[7] I. Abdul-Khalek, D. Kittelson, F. Brear, "The Influence of Dilution Conditions on Diesel Exhaust Particle Size Distribution measurements,” SAE Technical Paper Series, 1999-01-1142 ,1999. 